\title{
Intelligent Fault Diagnosis Based on ANN: A Review
}

\author{
Haipeng Pan \\ Institute of Automation, \\ Zhejiang Sci-Tech University \\ Hangzhou, 310018, China
}

\begin{abstract}
An overview of the fault diagnosis method based on Neural Network is presented. Some models based on neural network for fault diagnosis are discussed, and the problems existing in the fault diagnosis technology and the key study direction are also pointed out.
\end{abstract}

\section{Keywords-Fault diagnosis, Computational Intelligence, Neural network}

\section{INTRODUCTION}

With the increasing system complexity, the need to increase reliability and to decrease possible loss of production, efficient incipient faults detection and accurate faults diagnosis have been become critical in modern industrial systems. Fault detection and diagnosis is the process to detect, isolate, and identify faults in a system. Fault diagnosis can be viewed as the process of linking symptoms to causes, paralleling the field of medical diagnosis. Thus, the goal of fault diagnosis is to match the symptoms to the specific causes. Actually, the process of fault diagnosis is essentially a kind of recognition classification or recognition.

Artificial intelligence techniques, such as expert systems, artificial neural networks (ANN), fuzzy logic systems, and genetic algorithms etc. have been employed to assist the diagnosis. ANN has gained popularity over other techniques, as it is e cient in discovering similarities among large bodies of data, to learn the certain status or operation condition of the objective systems. And then the well-trained network can recognize these various conditions.

In this paper, methods of research in intelligent fault diagnosis based on neural network is reviewed.

\section{THE BASIC MODEL}

Algorithms is the core of the faults intelligent diagnosis, at present the neural network algorithm is a mature algorithm relatively, it includes feed-forward neural network, feedback neural network. Etc .

\section{A. Based on feedforward Neural Network}

1) Approach: Feedforward Neural Network has gained increasing attention recently for its simple structure, easy computing and programming. In 1989, K.Chan applied BPneural network in fault diagnosis of catalyst cracking apparatus, and satisfied results are achieved. BP neural network is a typical of teacher supervised learning network, whose learning process includes two phases.

2) Applications: In paper [1], an algorithm to detect and diagnose the fault with two classes of neural networks for

\author{
Bo Chen \\ Institute of Automation, \\ Zhejiang Sci-Tech University \\ Hangzhou, 310018, China
}

the fault in observations of dynamic precise point positioning occurs was presented. The first neural network training samples online is used to improve the reliability of dynamic model. The second neural network can automatically detect failures, position and delete the fault of observations, and the observations processed can further improve the contribution of dynamic model to the result of navigation.

3) Issues: BP Algorithm has some shortcomings, such as slow convergence rate, easily trapped into the local minimum during the training process, which results that it can't get the optimal solution, even misjudging in device fault diagnosis, though it is widely used.

\section{B. Based on feedback Neural Network}

1) Approach: In 1989, Professor Hopfield proposed a hopfield neural network (HNN) model that successfully solving the most representative travelling salesman problem. The output of each node in Hopfield neural network feeds back to other nodes' as their input. With the stirring of the network's input, the Hopfield neural network's state is changed continuously. If the variety of outputs gets smaller and smaller in each feedback and iteration computation process, the network converges to a stable state.

2) Applications: In paper [2], a Hopfield-BP neural network fault diagnosis method was proposed, which combined Hopfield neural network, having the global optimal neural network computing ability, with the BP neural network, charactering the nonlinear classification ability. It avoids the network to be trapped to a local optimum. Implementing the new network into the fault diagnosis of centrifugal fan has proven that fault pattern recognition could be solved well, and the accuracy of fault diagnosis is increased than that with the method of BP neural network.

3) Issues: The major advantage of $\mathrm{HNN}$ is in its structure can be realized on an electronic circuit, for an online solver with a parallel-distributed process. Although $\mathrm{HNN}$ is the most popular model of NNs for optimization, it suffers from some drawbacks [3]: (i) the minimal solution doesnot guarantee global optimization; (ii) the artificial variables are not easily implemented; and (iii) the energy function may pose many unnecessary local solutions which are difficult to avoid.

\section{Based on Self-Organizing Neural Network}

1) Approach: Self-organizing competitive neural network is composed of input layer and competitive layer. The network is based on unsupervised training, so it does not need to know corresponding outputs of training patterns. 
The network learns and classifies input patterns, and the standard patterns of every class are stored in the rows of weight matrix [4].

2) Applications: To overcome the weakness of dependence on the accurate model, a method using selforganizing neural networks for aeroengine fault diagnosis is developed [5]. Because self-organizing neural networks can be trained to adjust their own structures and eventually identify the faults only with the sensed data, the precise engine model is not necessary. With the help of the data preprocession, which extracts the features of faults, the neural networks finally get the correct results of fault diagnosis.

3) Issues: This neural network does not have local minimum point and has better convergence rate. It does not require a lot of experiments to find out the best structure, algorithm and operating parameter of neural networks. It can classify patterns with self-organizing training and is applicable to the classification problems of large quantities of data that have clustering characteristic. This network is a more stable model with higher diagnosis accuracy and better generalization capability.

\section{Based on Quantum Neural Network (QNN)}

1) Approach: In 2005, the QNN model in which the quantum state is conveyed by the quantum phase is proposed by Kouda. The three-tier construction of the feedforward neural network is employed, the quantum neuron acts as the hidden layer neurons, and the output layer neuron activation function chooses linear function. Through adjusting the quantum phase shift parameters, the model can realize the smooth processing to the output errors of different fault state cluster analysis, and represent the association between the sample parameters information and the implied fault state in a way of activate state probability reasoning, thus the speed and accuracy of pattern recognition can be improved [6].

2) Applications: The paper [7] show that quantum neural network has the characteristic of inherent ambiguity, which can assign the uncertainty data to each fault mode reasonably, making the network with the features of high capacity, better robust and timesaving. Meanwhile it can identify the majority of the sample fault modes correctly.

3) Issues: QNN has a better recognition capability to sort two different types of signals with uncertainties or fuzziness, and to eliminate the wrong or lost judgment caused by incomplete information from single-signal source or effect of similar fault mode [8]. So it can realize a good learning and training effect in fault diagnosis.

\section{HYBRID MODEL}

Each approach contains its own strengths and weaknesses. Hybrid solutions combine techniques to produce improved diagnostic solutions by capitalizing on the advantages of the individual techniques.

\section{A. Fuzzy Logic and Neural Network}

Fuzzy reasoning is capable of dealing with imprecise and uncertain information, and neural networks are capable of learning from examples. In contrast to neural network, the fuzzy logic systems can give a very clear physical description of how the function approximation is performed. Most of the research work relating to fuzzy logic and diagnosis has occurred in the area of dynamic industrial processes and electronic systems. The algorithm is especially fit for the application situation that there is numerous cases but not a good expression mode by mathematics.

\section{B. Expert System and Neural Network}

Expert systems have explicit representations of knowledge which eases the modification and validation process of such systems, are able to generate explanations concerning their reasoning methodology, and can use deep knowledge to reason about novel events. However, they are unable to learn from experience, are hard to maintain if the knowledge base becomes extremely large, and require extensive computational time if a deep model of the process must be consulted. Neural networks are unable to explain their own reasoning methodology, incorrectly generalize novel faults if improperly trained and forget past training if retrained on new data.The integration of two fundamentally different diagnostic techniques into a single hybrid architecture system was used successfully to diagnose faults in various industry fields.

\section{Rough Sets and Neural Network}

Rough set theory is presented by Poland scientist Z.PawLak in 1982. In the rough set theory, the approximation region is determined through the indiscernible relations and classes. By the knowledge reduction, the redundant information is deleted and the classified knowledge rules are induced. The hybrid method integrates the ability of variable precision rough set on reduction of diagnosis information system and that of neural network for fault classification. It provided an effective tool to study imprecise data's analyse and reasoning, dig the relation among data, discover the latent knowledge.

\section{Wavelet Neural Network (WNN)}

Wavelet analysis, originally developed from the Fourier transform at the end of 1980 s, is widely used in various engineering systems. Since it is capable of data processing, it can be used as the complement for neural network [10]. The essence of WNN is to find a family of wavelet in the characteristic space so that the complex function relationship contained in the original signal might be exactly expressed. The network has advantages of the wavelet transform in denoising, background deduction and recovery of characteristic information.

\section{E. Case-Based Reasoning and Neural Network}

Case-Based Reasoning (CBR) has been introduced in the early 1980s as a plausible reasoning approach supported by the idea that people reply on concrete previous experiences when solving new problems. When a new problem occurs, it will be represented as a new case and compared to the cases in the case base. Thus, the cases 
similar to the new cases will be used to suggest to users a solution for the new problem. Usually, the solved new case will also be added into the case base. However, CBR has some disadvantages. For example, previous cases may influence a CBR system in different directions without giving it many hints on which cases to consider as more important. By combining CBR with ANNs, we can benefit both from the logic-based and cognitive nature of symbolic systems and from the numeric, associative and self-adapting nature of ANNs [11].

\section{The Optimization Model}

\section{A. Neural Network optimized by Genetic Algorithm}

The genetic algorithm (GA) developed by John Holland in 1975 is a particular class of evolutionary algorithms well known for optimization of nonlinear functions. They use a structure similar to a chromosome and apply operators of selection and crossing in order to preserve information relevant to the problem solution. The GA belongs to the class of probabilistic search and optimization methods, although it is not merely a random search. Requires only information about the objective function to be optimized and evaluated for each population member. GA is used to select the most significant features from the whole feature database and optimize the ANN structure parameter. This combination reduces the learning time and increases the diagnosis accuracy.

\section{B. Neural Network optimized by Particle Swarm Optimization}

The combination of the Particles Algorithm and the Neural Network is based on overcoming the lower reliability in the study of the Neural Network and the global search strategy. Particle swarm optimization (PSO), first introduced by Kennedy and Eberhart, is one of the modern heuristic algorithms. It was developed through simulation of a simplified social system, and has been found to be robust in solving continuous nonlinear optimization problems. The PSO technique can generate a high-quality solution within shorter calculation time and stable convergence characteristic than other stochastic methods. PSO algorithm is mainly used to train the neural network for getting the weight and bias.

\section{Neural Network optimized by Ant Colony Algorithm}

The ant colony optimization (ACA), as a kind of stochastic search algorithm proposed by Dorigo, is inspired by the behavior of real ant colony, which can find a shortest path from the nest to a food source. The optimal solution is gained according to the shortest path of ant tours. Ant colony optimization is a powerful and adaptable tool for parameter optimization.

However, the search efficiency and convergence speed of ant colony algorithm are low and algorithm tends to trap in local optimum.

\section{Neural Network optimized by Immune Algorithm}

Artificial Immune System (AIS) is an intelligent way to imitate the natural function of the immune system, it implements a biological immune system illumination, by learning the natural defense mechanism's learning technology of outside materials, it provides learning mechanism of evolution, such as noise patience, learning without teachers, self-organizing, memory and so on. Immune algorithm and neural network algorithms are established which are inspired by the information handling manners of the biological systems, there are a lot of similarities in their manners of data handling, which can be combined with each other to explore a mixed algorithm of the artificial immune and BP hybrid neural network [12].

The main idea of the immune neural network diagnosis method is to optimize the weights of the neural network globally using immune algorithm firstly utilizing its characteristics of preservation of diversity and global convergence [13]. The hybrid algorithm can improve the accuracy and speed of fault diagnosis.

\section{FUTURE DEVELOPMENT}

The purpose of fault diagnosis is to improve the accuracy and speed, reduce the rate of missing report, and estimate the size and trend of the fault. With the rapid development of artificial intelligence in the diagnosis of further application, the main future research can be summarized as the following aspects.

1) Multi-information Fusion. Fault diagnosis based on multi-sensor data fusion can make the most of the information of multi-sensor and identify faults which cannot be recognized with single sensor data, and that fault without obvious early features can be found as soon as possible and identified accurately by means of multi-sensor data fusion.

2) Hybrid System. The drawbacks of single-method based diagnostic systems are serious enough to limit their applications to small case studies and render them unsuitable for large-scale control systems.

3) Network Diagnostic Techniques. Now, setting up WWW Based Remote Fault Diagnosis System by combining equipment fault diagnosis technology and Internet technology is available. And it is an urgency which every modern enterprises is confronted with that how to integrated the data net with control net.

4) Adaptive Intelligent Fault Diagnosis. CompoundAgent can be used to construct the system with selfadaptability, rapider response and more intelligence, which adapts to the complex dynamic giant system better.

\section{SUMMARIES}

This paper has given a general review of the recent developments in the field of AI-based diagnostic systems .To conclude, having been developed for many years, the theories and technologies of fault diagnosis have advanced greatly but those achievements are far from the solution to all problems, farther research and development in both of them must be made. 


\section{CKNOWLEDGMENT}

This project is supported by National Natural Science, Fund (No: Y1110686).

\section{REFERENCES}

[1] NIE Jianliang; GAO Weiguang; ZHANG Shuangcheng. "Detection and Diagnosis of Failures with BP Neural Network in Dynamic Precise Point Positioning," Geomatics and Information Science of Wuhan University [J], 2010,35(3), pp. 283-285.

[2] Hong Rao; Meizhu Li; Mingfu Fu. "Equipment diagnosis method based on hopfield-BP neural networks," International Conference on Advanced Computer Theory and Engineering, ICACTE 2008 [C], pp 170-173.

[3] Ue-Pyng Wena; Kuen-MingLana; Hsu-Shih Shih. "A review of Hopfield neural networks for solving mathematical programming problems," European Journal of Operational Research [J]. 2009,198(3), pp. 675-687.

[4] Peng Xua; Shijin Xua; Hongwei Yin. "Application of self-organizing competitive neural network in fault diagnosis of suck rod pumping system," Journal of Petroleum Science and Engineering [J]. 2007, 58(1), pp. 43-48.

[5] CHEN Tian, SUN Jian-guo etc. "Self-Organizing Neural Network Based Fault Diagnosis for Aeroengine Gas Path," Acta Aeronautica et Astronautica Sinica [J], 2003, 24(1), pp. 46-48.
[6] Cai Guowei; Liu Ning; Yang Deyou; "The Transformer Fault Diagnosis Based on Quantum Neural Network," Computer, Mechatronics, Control and Electronic Engineering (CMCE), 2010 International Conference [C].2010, pp. 396-400.

[7] Long, Bohua;Tan, Yanghong. "Fault Diagnosis of Power Electronic Circuits Based on Quantum Neural Network," Diangong Jishu Xuebao [J]. 2009,24(10), pp. 170-175.

[8] Zhengyou He etc. "A New Power System Fault Diagnosis Method Based on Rough Set Theory and Quantum Neural Network," Power and Energy Engineering Conference 2009 [C]. pp. 1-4.

[9] Haiying Yuan; Guangju Chen. "Fault Diagnosis in Nonlinear Circuit Based on Volterra Series and Recurrent Neural Network," Neural Information Processing [J]. 2006, Vol4234, pp. 518-525.

[10] Zhimin Du; Xinqiao Jina. "Fault diagnosis for temperature, flow rate and pressure sensors in VAV systems using wavelet neural network," Applied Energy [J]. 2009, 80(9), pp. 1624-1631.

[11] Bo-Suk Yang;Tian Han. "Integration of ART-Kohonen neural network and case-based reasoning for intelligent fault diagnosis," Expert Systems with Applications [J]. 2004,26(3), pp. 387-395.

[12] Lian-Dong $\mathrm{Fu}$; Kui-Sheng Chen. "Application of immune neural network for electro-hydraulic servo valve fault diagnosis," Machine Learning and Cybernetics, 2008 International Conference [C].2008, pp. $1898-1902$.

[13] Zongyan Yu."Research of Fault Diagnosis Method Based on Immune Neural Network," WRI Global Congress on Intelligent Systems [C],2009,vol 02, pp. 69-73. 